\title{
Silver Nanoparticle Loaded Activated Carbon: An Escalated Nanocomposite with Antimicrobial Property
}

\author{
C. KARTHIK ${ }^{1 *}$ and K. V. RADHA ${ }^{2}$ \\ 'Department of Biotechnology, St. Joseph's College of Engineering, \\ Old Mamallapuram Road, Chennai-600 119, Tamil Nadu, India. \\ 2Department of Chemical Engineering, A. C. College of Technology, Anna University, \\ Chennai- 600 025, Tamil Nadu, India. \\ ${ }^{*}$ Corresponding author E-mail: karthikc.bio@ gmail.com \\ http://dx.doi.org/10.13005/ojc/320182
}

(Received: May 01, 2015; Accepted: January 02, 2016)

\begin{abstract}
In this work, the antimicrobial property of nanocomposite prepared by loading of silver nanoparticle onto activated carbon (AgNP-AC) was studied. The silver nanoparticles were synthesized biologically from the culture Enterobacter aerogenes and loaded onto the activated carbon. The nanoparticle and nanocomposite were characterized by UV-vis, scanning electron microscopy and X-ray diffraction studies. The spectroscopic assay and zone inhibition test for $E$. coli and $B$. subtilis clearly illustrates the antibacterial activity of the silver nanoparticle and nanocomposite. The antimicrobial activity of the nanocomposite was of the nanocomposite was also confirmed with the observed zone of inhibition against beverage, brewery, dairy and dye industrial effluents.
\end{abstract}

Key words: Silver Nanoparticle, Activated Carbon, AgNP-AC Composite, Antibacterial activity.

\section{INTRODUCTION}

Many methods have been adopted for treatment of waste water and industry effluents. One of the best and convenient water treatment process is by adsorption, as it is cheap, easy and simple in operation ${ }^{1}$. The most popular and commonly used adsorbent in water and wastewater treatment throughout the world is activated carbon $(\mathrm{AC})^{2}$. AC as adsorbent have relatively high surface area, large porosity, total pore volume and presence of wide spectrum of functional groups on its surface, which provides a strong affinity for even low concentration organics to attach to itself ${ }^{3}$. The well developed internal pore structure of $\mathrm{AC}$ with macropores serves as excellent loci for colonization of organisms and support material for bacterial growth ${ }^{4}$. The biofilm layer formations on the AC by the microbes have undesirable effects: the filter may clog up as a result of excessive bacterial growth. Nowadays, nanoparticles are used as sorbents for organic and inorganic pollutants removal because of their high specific surface area and also a large number of unsaturated atoms on their surfaces that can bind readily with most of other atoms ${ }^{5,6,7}$. More attention is focused to water treatment with 
engineered nanoparticles ${ }^{8}$. These nanoparticles can be produced by numerous techniques, including chemical, aerosol, electrochemical, laser irradiation, sonochemical deposition, photochemical reduction and biological techniques ${ }^{9,27}$. The biotechnological experimental processes gains importance because of the advantages like safe, cost-effective, sustainable and environmentally friendly processes ${ }^{10}$. The nanoparticles has very attractive properties such as an ordered structure with a high aspect ratio, ultralight weight, high mechanical strength, electrical and thermal conductivity and high specific surface area. The nanoparticles property differs from those of their corresponding bulk state ${ }^{11}$. Among the nanoparticles, silver nanoparticles (AgNP) are gaining more importance because of its antimicrobial and antiviral properties $^{12}$. The believed mechanism is that AgNPs attach to the surface of cell membrane disturbing the permeability and respiration functions of the cells, which leads to microbial cell death ${ }^{13}$. Nanocomposite as an adsorbent will prove as an efficient adsorbent because of the increase in their surface-to-volume ratio with the reduction of the size of the adsorbent particle from bulk to nano dimensions ${ }^{14}$. In the present study, silver nanoparticles were biogenically synthesized using Enterobacter aerogenes and the synthesized nanoparticles were incorporated onto activated carbon to produce a nanocomposite. These nanoparticles and nanocomposites were characterized using UV-vis spectroscopy, SEM and $X R D$. Further the antimicrobial activity of silver nanoparticles and nanocomposites were studied against E. Coli, B. Subtilis and microbes present in the various industrial effluents like beverage, brewery, dairy and dye respectively.

\section{MATERIALS AND METHODS}

All chemicals used in the analysis are of analytical grade and were procured from Sigma Aldrich \& Hi Pure chemicals, India and did not require any further purification. The culture Enterobacter aerogenes (MTCC 111) was obtained from Microbial Type Culture Center (MTCC), Chandigarh, India.

\section{Synthesis of AgNP}

In $100 \mathrm{ml}$ of $1 \mathrm{mM}$ silver nitrate $\left(\mathrm{AgNO}_{3}\right)$ solution, $5 \%(\mathrm{v} / \mathrm{v})$ of Enterobacter species culture supernatant sample was added slowly and was observed for colour change. The particles resulting from the reduction of $\mathrm{AgNO}_{3}$ are called as AgNP.

\section{Preparation of Nanocomposite}

The AgNP was loaded on AC by means of simple mixing. $1.5 \mathrm{~g}$ of $\mathrm{AC}$ was taken and added to result AgNPs solution. It was mixed vigorously by continuous stirring for $2 \mathrm{~h}$ at $150 \mathrm{rpm}$ using Meditek Orbital rotary double deck shaker and Ultrasound radiating the solution for $40 \mathrm{~min}$ at 100 $\mathrm{Hz}$ using Ultrasonic Processor P2 (Vibronics). The nanocomposite product was obtained by drying the AgNP loaded activated carbon (AgNP-AC) powder in air at a temperature of $75^{\circ} \mathrm{C}$.

\section{Characterization studies}

The Surface plasmon resonance (SPR) property of the synthesized AgNP was measured using Systronics UV-vis double beam spectrophotometer 2202. The morphological, size and elemental characterization of the normal activated carbon and the AgNP-AC was carried out using a FEI Quanta FEG 200 FESEM at an operating voltage of $10 \mathrm{kV}$. The XRD pattern of the AC and Ag-NP was determined using Spectris Technologies X'pert Powder X-ray diffractometer with Cu Ká radiation (40 $\mathrm{kV}$ and $30 \mathrm{~mA}$ ) for 2è values over $4-100^{\circ} \mathrm{C}$.

\section{Antimicrobial studies \\ AgNP - Direct method}

The antibacterial property of AgNP was determined using zone inhibition test and indirect growth curve method (cell number versus time of incubation) for $E$. coli and B. subtilis. For zone inhibition test the $100 \mu$ l of the organisms E. coli and B. subtilis grown overnight in Luria broth (LB) medium was spread on a nutrient agar plate. The plates were then punctured and fed with a colloidal AgNP solution and incubated at $37^{\circ} \mathrm{C}$. The zone of inhibition was then measured after $24 \mathrm{~h}$ of incubation.

\section{AgNP -Indirect method}

LB media was prepared in a sterile manner and $100 \mathrm{ml}$ of the media was poured into flasks labeled as A, B, C \&D. A $100 \mathrm{ml}$ of E. coli and B. subtilis bacterial suspension was inoculated into flasks A, B \& C, D respectively. $1 \mathrm{ml}$ of the resultant AgNP from $5 \mathrm{mM} \mathrm{AgNO}_{3}$ solution was added into flasks B and D. Flasks A \& C was kept as control, 
while $B$ \& $D$ as the test for E. coli and B. subtilis, accordingly. The flasks are incubated in a rotary shaker at $37^{\circ} \mathrm{C}$. The growth was monitored for the control and the test by withdrawing aliquots of control and test samples for every $20 \mathrm{~min}$ and measuring the absorbance at $600 \mathrm{~nm}$ using a Mapada V-1100 D spectrophotometer. A graph was plotted for time in min (abscissa) Versus OD at $600 \mathrm{~nm}$ (ordinate).

\section{Nanocomposite}

The antibacterial property of the nanocomposite was evaluated using zone inhibition method. A $100 \mathrm{ml}$ of $E$. coli and B. subtilis bacterial suspension cultured in LB medium was spread on a nutrient agar plate. The plates were then punctured and loaded with normal activated carbon (control) and AgNP-AC (Test) to find the antibacterial properties; the plates were then incubated at $37^{\circ} \mathrm{C}$. The zones of inhibition were measured after $24 \mathrm{~h}$.

\section{Industrial Effluents}

The antibacterial property of the nanocomposite was evaluated using zone inhibition method. A $250 \mu$ l of beverage, brewery, dairy and dye industrial effluents were spread on a nutrient agar plate. The plates were then punctured and loaded with normal activated carbon (control) and AgNP-AC (Test) to find the antimicrobial properties; the plates were then incubated at $37^{\circ} \mathrm{C}$. The zones of inhibition were measured after $24 \mathrm{~h}$.

\section{RESULTS AND DISCUSSION}

\section{Extracellular production of AgNPs}

The $\mathrm{AgNO}_{3}$ solution was reduced to AgNPs due to the addition of the supernatant containing extracellular material produced by Enterobacter species. Fig. 1 shows the change of colour from colourless to yellow-brownish, which clearly indicates the formation of AgNPs in the reaction mixture. The characteristics colour of the colloidal silver solution is due to the excitation of surface plasmon vibrations in the nanoparticle and provides a convenient spectroscopic signature of their formation. The principle behind this process is the

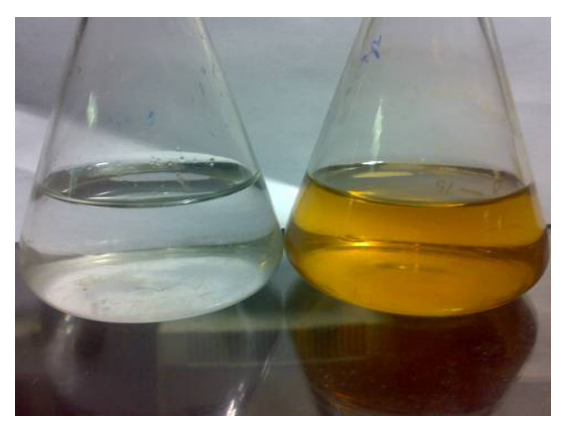

Fig. 1: Colour change of silver nitrate $1 \mathrm{mM}$ solution from colourless to yellow brownish as the reaction proceeds on addition of the culture supernatant

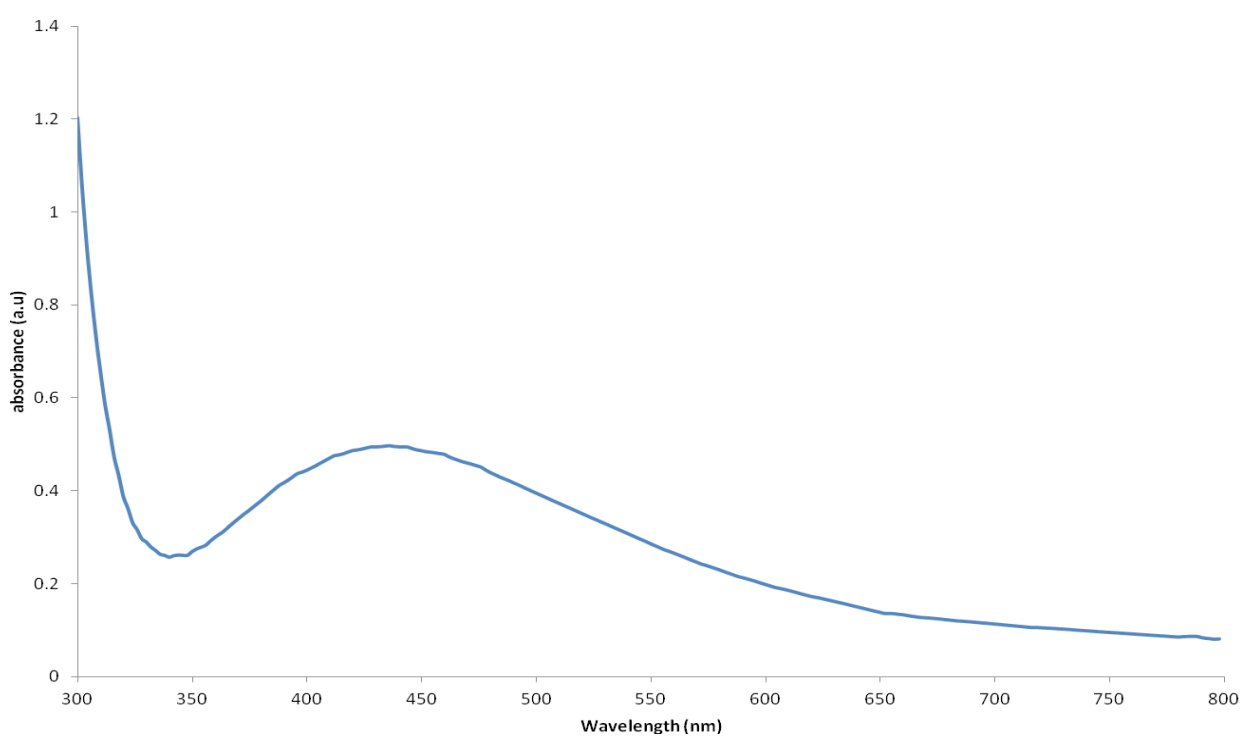

Fig. 2: UV-visible spectra recorded from the aqueous silver nitrate-culture supernatant reaction medium. 
activity of a most abundantly found enzyme nitrate reductase which reduces nitrate to nitrite and thereby reducing $\mathrm{Ag}^{+}$ions to $\mathrm{AgNPs}$. Several hydroquinones with excellent redox properties were reported that could act as electron shuttle in metal reductions. Thus, it was evident that electron shuttle or other reducing agents released by Enterobacter species are capable of reducing silver ions to $\mathrm{AgNPs}^{15,16,17}$

\section{SPR of AgNP}

The AgNPs were characterized using UV-visible spectroscopy, the results is shown in Fig. 2. A strong, broad peak located in 410-420 $\mathrm{nm}$ was observed for the AgNPs prepared using the Enterobacter species. Observation of this peak assigned to a surface plasmon is a strong evident for the formation of silver metal nanoparticles ${ }^{18,19,20}$.

\section{Morphological Characterization Silver Nanoparticle}

Fig. 3(a) shows the SEM micrograph recorded for AgNP range from 25-85 $\mathrm{nm}$. The morphology of the nanoparticles was found to be spherical, monodispersed and uniformly distributed. The particles were not aggregated, which might be an indication for the presence of caping agent. The SEM images for AC and AgNP-AC were shown in Fig. 3 (b) \&3 (c) respectively. AC was in the range of 125-185 nm and AgNP in nanocomposites were recognized as bright dots, which confirms the incorporation process was successful.

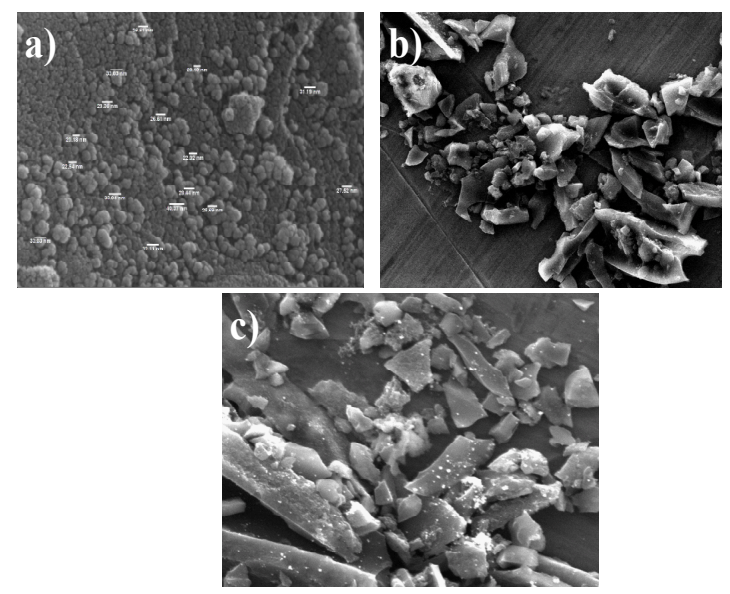

Fig. 3: SEM micrograph: a) AgNP b)Activated Carbon c) Nanocomposite (AgNP-AC)

\section{XRD Pattern}

The XRD patterns of AC and AgNP-AC are shown in Fig. 4. The broad peaks of $A C$ and the sharp peaks of silver appeared in the results of the AgNP-AC display the variations occur in both AgNP$A C$ and $A C$. The XRD pattern of AC did not show any diffraction peak indicating that it is disordered (Fig. $4 A)^{5}$. As shown in Fig. 4 (B), the pattern of AgNP-AC exhibits peaks at peaks at $2 \theta$ angles of $38.15^{\circ}, 46.5^{\circ}$, $64.5^{\circ}$ which corresponds to AgNP21.

\section{Antimicrobial property Study \\ Silver nanoparticle - Direct method}

Antibacterial test by zone of inhibition was done to qualitatively determine the level of inhibition using the synthesized colloidal AgNP. Fig. 5 shows the antibacterial zone of AgNP for E. coli (Fig. 5a) and $B$. subtilis (Fig. 5b). For both the organisms the zone of inhibition was $1.7 \mathrm{~cm}$, which correlates with the antibacterial property of the synthesized AgNP 22,23 .

\section{Silver nanoparticle - Indirect method}

The Fig. 6 ( $a$ \& b) depicts the growth curve pattern of $E$. coli and $B$. subtilis at $37^{\circ} \mathrm{C}$ respectively, which act as an empirical model of the evolution of a quantity over time. The graphs nature for the test over the control in both cases shows that the AgNP added into the medium of $E$. coliand $B$. subtilis clearly acts as an antibacterial agent. The AgNP into the medium has clearly inhibited the growth of $E$. coli

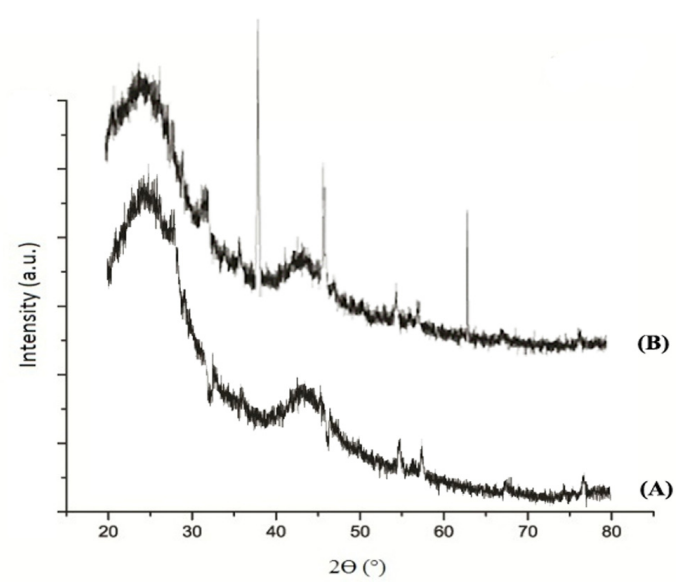

Fig. 4: XRD pattern: (A) Activated Carbon (B) AgNP-AC 
and $B$. subtilis by more than $90 \%$. The inhibition may be due to the penetration of the nanoparticle into the cell membrane and causing structural changes and thereby resulting in death or by free radical generation by the AgNP24.

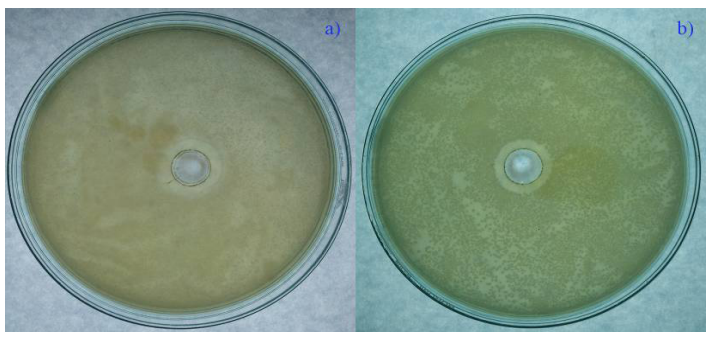

Fig. 5: Bacteria Grown on agar plates loaded with AgNP: a) E. coli b) B. subtilis

\section{Nanocomposite}

Fig. 7 (a \& b) confirmed that the AgNP has antibacterial activity against $E$. coli and $B$. subtilis. In both the cases the control $(A)$ : only activated carbon did not show any inhibition, but the test $(B)$ : AgNP-AC significantly showed zone of inhibition of 1.8 and $1.7 \mathrm{~cm}$ for $E$. coli and $B$. subtilis respectively. The zone of inhibition of the test shows that the nanoparticles of silver were well incorporated onto the activated carbon and thereby responsible for the antibacterial activity ${ }^{25}$. The nanoparticles introduction to the activated carbon also decreases the pore volumes and surface area ${ }^{26}$. We suggest that the nanoparticle incorporated activated carbon can be used as an efficient adsorbent instead of normal activated carbon, as the nanoparticles incorporated onto activated carbon will influence the growth of

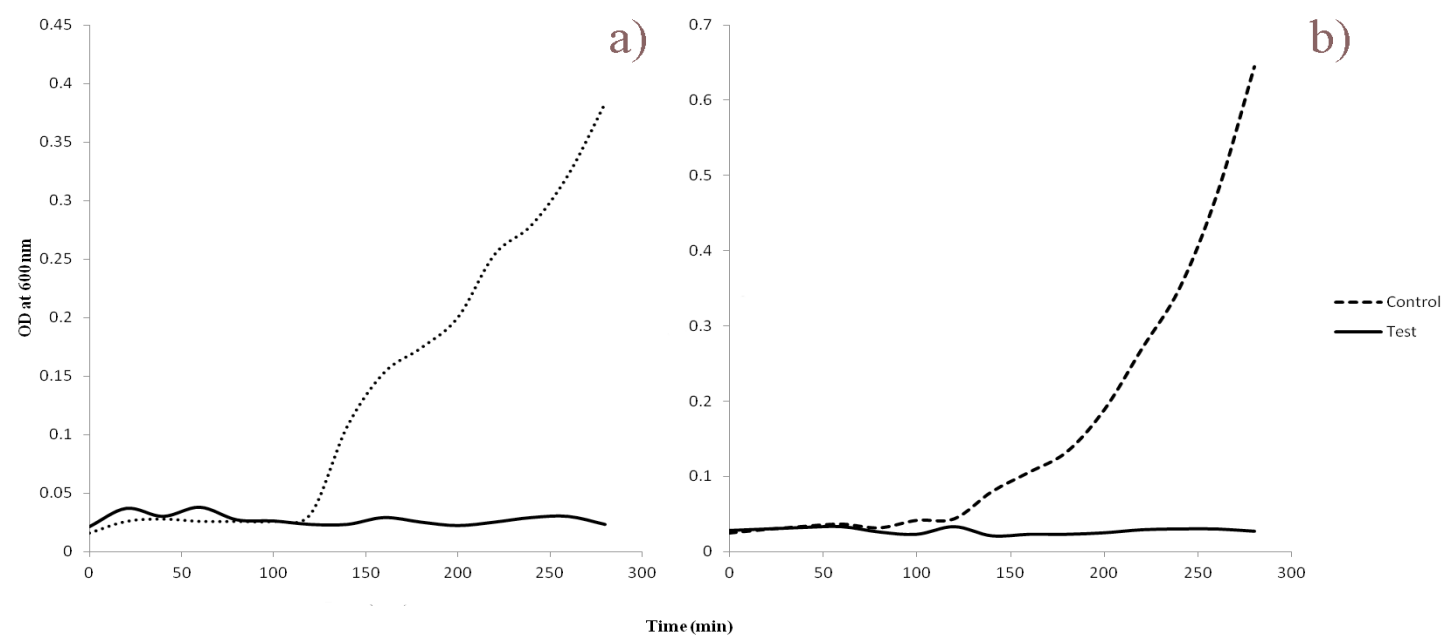

Fig. 6: Growth curve pattern: a) E. coli b) B. subtilis

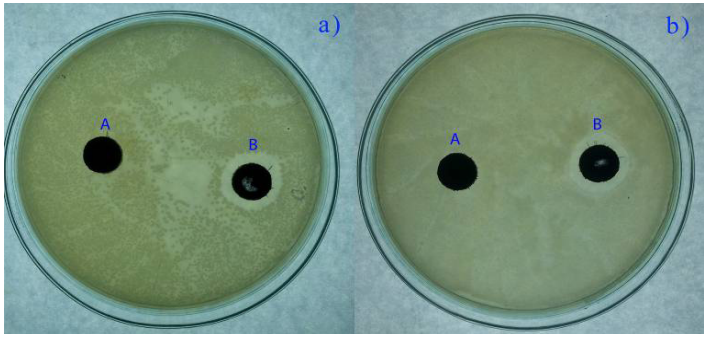

Fig. 7. Bacteria grown on agar plates with activated carbon as control $(A)$ and nanocomposite as test (B) : a) E. coli b) $B$. subtilis
Table 1: Antibacterial activity of silver nanoparticles loaded activated carbon nanocomposite for the industrial effluents incubated at $37^{\circ} \mathrm{C}$ for $24 \mathrm{~h}$

\begin{tabular}{llcc}
$\begin{array}{l}\text { S. } \\
\text { No }\end{array}$ & Industrial Effluent & $\begin{array}{c}\text { Zone of } \\
\text { Inhibition }\end{array}$ & $\begin{array}{c}\text { Diameter } \\
(\mathbf{m m})\end{array}$ \\
\hline 1. & Brewery Effluent & + & 18 \\
2. & Dairy Effluent & + & 20 \\
3. & Dye Effluent & + & 16 \\
4. & Beverage Effluent & + & 18 \\
\hline
\end{tabular}


bacteria and does not act as a support for biofilm layer formation.

\section{Antimicrobial Test for Industrial Effluents}

Antibacterial test by zone of inhibition was done to qualitatively determine the level of inhibition using the synthesized AgNP-AC nanocomposite for the four effluents were shown in Table 1. For all the industrial effluents an appreciable level of zone of inhibition was observed, the zone of inhibition was $18 \mathrm{~mm}, 20 \mathrm{~mm}, 16 \mathrm{~mm}$ and $18 \mathrm{~mm}$ for brewery, dairy, beverage and dye effluents accordingly. The results clearly shows that the AgNP loaded activated carbon nanocomposite possess the antibacterial activity. This property of the prepared nanocomposite adsorbent will prevent the bacterial biofilm formation over the adsorbent and increases the possible reuse of the adsorbent in the waste water treatment processes, without loss in adsorption property.

\section{CONCLUSION}

Silver nanoparticles were synthesized successfully by a simple biogenic route. The synthesized nanoparticles were characterized using UV-vis spectroscopy, SEM and XRD. The incorporation of synthesized AgNP onto activated carbon was done and the nanocomposite was prepared and confirmed. The growth curve estimation method for $E$. coli and $B$. subtilis shows that the synthesized AgNP inhibits the microbial growth proving its antibacterial property. Zone inhibition test showed that the AgNP-AC is possessing antibacterial activity to that of normal activated carbon, which provides the possible use in waste water treatment process preventing the biofilm layer growth over the adsorbent. Finally, silver nanoparticle loaded activated carbon will be a desirable adsorbent in water treatment process.

\section{REFERENCES}

1. Bhatnagar, A.; Vilar, V. J. P.; Cidalia M.S.; Rui, B.; Boaventura, A.R., Adv. Colloid Interface Sci. 2010, 160, 1-15.

2. Bhatnagar, A.; Hogland, W.; Marques, M.; Sillanpaa, M., Chem. Eng. J. 2013, 219, 499511.

3. Ahmad, M. A.; Alrozi, R.. Chem. Eng. J. 2011, 171, 510-516.

4. Kalkan, C.; Yapsakli, K.; Mertoglu, B.; Tufan, D., Desalination. 2011, 265, 266-273.

5. Ghaedi. M.; Karimi. F.; Barazesh. B.; Sahraei. R.; Daneshfar., J. Ind. Eng. Chem. 2013, 19, 756-763.

6. Tuan,T. Q.; Son, N. V.; Dung, H. T.K.; Luong, N. H.; Thuy, B. T.; Anh, N. T. V.; Hoa, N. D.; Hai, N. H., J. Hazard. Mater. 2011, 192, 1321-1329.

7. Sandoval, R.; Cooper, A.M.; Aymar, K.; Jain, A.; Hristovski, K., J. Hazard. Mater. 2011, 193, 296-303.

8. Xu, J. H.; Gao, NY.; Deng, Y.; Xia, S. Q., Chem. Eng. J. 2013, 222, 520-526.

9. Shahverdi, A.R.; Minaeian, S.; Shahverdi, H.R.; Jamalifar, H.; Nohi, A.: A novel approach, Process Biochem. 2007, 42, 919-923.

10. Sivaraman, S.K.; Elango, I.; Kumar, S.; Santhanam, V.. Curr. Sci. 2009, 97, 10551058.
11. Ghaedi M.; Sadeghian B.; Amiri Pebdani A.; Sahraei R.; Daneshfar A.; Duran C., Chem. Eng. J. 2012, 187, 133-141.

12. Chen, Y.; Chen, H.; Zheng, X.; Mu, H.,J. Hazard. Mater. 2012, 239-240, 88-94

13. Anandhakumar, S.; Raichur, A.M., Colloids Surf. B. 2011, 84, 379-383.

14. Ghaedi, M.; Ghayedi, M.; Kokhdan, S. N.; Sahraei, R.; Daneshfar, A., J. Ind. Eng. Chem. 2013, 19, 1209-1217.

15. Saifuddin, N.; Wong, C. W.; Nur Yasumira, A. A., E-J. Chem. 2009, 6, 61-70.

16. Minaeian, S.; Shahverdi, A. R.; Nohi, A. S.; Shahverdi, H. R., J. Sci. I. A. U. 2008 , 17.

17. Kasthuri, J.; Verrapandian, S.; Rajendiran, N., Colloids Surf. B. 2009, 68, 55-60.

18. Fayaz, A. M.; Balaji, K.; Kalaichelvan, P. T.; Venkatesan, R., Colloids Surf. B. 2009, 74, 123-126.

19. Sadowski, Z.; Maliszewska, I. H.; Grochowalska, B.; Polowczyk, I.; Kozlecki, T., Mater. Sci-Poland. 2008, 26, 419-424.

20. Sharma,V. K.; Yngard, R. A.; Yekaterina, Lin., Adv. Colloid Interface Sci. 2009, 145, 83-96.

21. Flores, C.Y.; Diaz, C.; Rubert, A.; Benítez, G.A.; Moreno, M.S.; Fernández Lorenzo de 
Mele M.A.; Salvarezzaa, R.C.; Schilardi, P.L.; Vericat, C., Chem. Eng. J. 2012, 187, 133141.

22. Guzman, M.; Dille, J.; Godet, S., Nanomed. Nanotech. Biol. Med. 2012, 8, 37-45.

23. Manikprabhu, D.; Lingappa, K., J. Pharm. Res. 2013, 6, 255-260.

24. Prabhu, S.; Poulose, E, K. In. Nano Lett. 2012, $2: 32$.
25. Hsu, S.; Tseng, H.; Lin,Y., Biomaterials. 2010, 31, 6796-6808.

26. Bashkova, S.; Deoki, D.; Bandosz, T. J. J. Colloid. Interf. Sci. 2011, 354, 331-340.

27. Raj, LFAA.; Jayalakshmy, E.. Orient. J. Chem. 2015, 31, 51-56.

28. Ghorbani, H. R.; Mehr, F. P.; Poor, A. K.. Orient. J. Chem. 2015, 31, (1),527-529.

29. Maleknia, L.; Rashidi, A. S.; Ghamsari, N.L.,. Orient. J. Chem. 2015, 31(1), 257-262. 